\title{
CARACTERISTICAS DO CUIDADO A MULHERES COM TRANSTORNOS MENTAIS NA ATENÇÃO BÁSICA PARA CONSTRUÇÃO DE GESTÃO DE CASO
}

\author{
CARE CHARACTERISTICS OF WOMEN WITH \\ MENTAL DISORDES IN THE BASIC ATTENTION FOR \\ THE CONSTRUCTION OF CASE MANAGEMENT
}

Michele Cecilia Silva Torréziol

Nadja Cristiane Lappann Botti ${ }^{2}$

\begin{abstract}
RESUMO
Objetivo: Este estudo teve como objetivo descrever o perfil e caracterizar o atendimento a mulheres com transtornos mentais pela Estratégia de Saúde da Família (ESF). Método: Estudo de abordagem qualitativa, realizado através da análise de prontuários de atendimentos de pacientes pertencentes à ESF da cidade de Divinópolis/MG, entre outubro de 2014 a março de 2015. Resultados/Discussão: Todas as pacientes faziam uso de algum tipo de medicamento para tratamento de morbidade clínica ou psiquiátrica, sendo a classe de ansiolíticos e antidepressivos os mais prescritos. Todas as mulheres foram atendidas na Atenção Básica, sendo o atendimento de livre demanda mais frequente que o atendimento agendado. Apesar de, a Atenção Básica ser a principal forma de entrada na rede de saúde, a continuidade do cuidado a essas pacientes não ficou evidenciado. Consideraçóes finais: Existe a necessidade da criação de estratégias mais específicas, que visam promover a continuidade do acompanhamento e tratamento dos pacientes.
\end{abstract}

Palavras-chave: Saúde Mental. Assistência à Saúde. Saúde da Família. Administração de Caso.

\begin{abstract}
Objective: The aim of this study is to describe the profile and characterize the service of women with mental disorder in the Family Health Program (ESF). Method: Qualitative study accomplish by analyzing calls of medical records of patients in the ESF of Divinópolis City in Minas Gerais, between October, 2014 and March, 2015. Results and discussion: All the patients used some kind of drugs for the treatment to the clinical morbidity or psychiatric morbidity being the class of anxiolytic and antidepressant more prescriptions. All the women wereattended in Primary Health Care, being the patients without referrals more frequent than programmed care. Despite that Primary Health Care be the principal way to in health service, the continuity of care with these patients were not evident. Final considerations: There is need to create more specific strategy that want to promote the continuity of care and treatment of patients.
\end{abstract}

Keywords: Mental Health. Health Care. Family Health. Case Management.

Enfermeira. Especialista em Saúde da Família pelo Programa de Residência da Universidade Federal de São João del Rei (UFSJ) Campus CentroOeste Dona Lindu.

Enfermeira. Psicóloga. Doutora em Enfermagem Psiquiátrica. Professora Associada I da Universidade Federal de São João del Rei (UFSJ) Campus Centro-Oeste Dona Lindu. 


\section{INTRODUÇÃO}

A Atenção Básica tem como um de seus princípios possibilitar o primeiro acesso das pessoas ao sistema de Saúde, inclusive aquelasque demandam um cuidado em saúde mental. É caracterizada por açóes em saúde de cunho individual e coletivo, que englobam intervençóes de promoção, proteção, prevenção de agravos, diagnóstico, tratamento, reabilitação, redução de danos e manutenção da saúde, sendo orientadas por conceitos como universalidade, acessibilidade, coordenação do cuidado, vínculo, integralidade, participação social e humanizaçãoo ${ }^{(1-2)}$.

A Política Nacional da Atenção Básica elegeu a Saúde da Família como a estratégia prioritária para a organização das açôes em saúde, nesse nível de atenção ${ }^{(3)}$. Corroborando com Correia, Barros e Colvero $^{(4)}$, as açōes de saúde mental na saúde da família devem fundamentar-se nos princípios do SUS e da Reforma Psiquiátrica. A desinstitucionalização, princípio da Reforma Psiquiátrica, pressupóe a manutenção do paciente com transtorno mental em seu cotidiano, evitando assim a internação, e possibilitando a preservação dos vínculos entre pacientes com seus familiares e suas redes sociais.

Neste sentido, Estevam et al. ${ }^{(5)}$ refere em seu estudo, que a Estratégia de Saúde da Família (ESF) tem o domicílio como espaço terapêutico e a assistência humanizada como instrumento facilitador para criação de vínculos e aproximação dos profissionais com o cotidiano das famílias. Dessa forma, a ESF tem potencial para o atendimento de pessoas com transtornos mentais, já que atua na área de abrangência de suas famílias.

Assim, as ações de saúde mental na atenção básica devem obedecer ao modelo de redes de cuidados, da base territorial a atuação transversal, com outras políticas específicas e que busquem o estabelecimento de vínculos e acolhimento ${ }^{(6)}$.

Assim, as Redes de Atenção à Saúde (RAS) são os arranjos organizativos de açôes e serviços, que gerenciam técnica, logística e gestão, buscando garantir a integralidade do cuidado. As RAS caracterizam-se: pela formação de relações entre os pontos de atenção com o centro de comunicação na Atenção Básica, pela centralidade nas necessidades em saúde da população, pela responsabilização na atenção contínua e integral, pelo cuidado multiprofissional, pelo compartilhamento de objetivos e compromissos com os resultados sanitários e de planejamento econômico ${ }^{(6)}$.

$O$ modo de organizar as RAS define a singularidade de seus processos descentralizadores frente a outros setores sociais. Os serviços de saúde estruturam-se numa rede de pontos de atenção à saúde, composta por equipamentos de diferentes densidades tecnológicas, que devem ser distribuídos, espacialmente, de forma ótima. A organização das RAS, para ser feita de forma efetiva, eficiente e com qualidade, tem de estruturar-se com base nos seguintes fundamentos: economia de escala, disponibilidade de recursos, qualidade e acesso, integração horizontal e vertical, processos de substituição, territórios sanitários e níveis de atenção ${ }^{(7)}$.

A Organização Mundial da Saúde considera que as RAS contêm seis modalidades de integração, e partir dessas modalidades produz uma conceituação de serviços integrados à saúde, como "a gestão e a oferta de serviços de saúde de forma a que as pessoas recebam um contínuo de serviços preventivos e curativos, de acordo com as suas necessidades, ao longo do tempo e por meio de diferentes níveis de atenção à saúde"(8).

Para Rosene $\mathrm{Ham}^{(9)}$, os objetivos de uma RAS são: melhorar a qualidade da atenção e a qualidade de vida das pessoas usuárias, os resultados sanitários do sistema de atenção à saúde, a eficiência na utilização dos recursos e a equidade em saúde. E dentre seus atributos estão: população e território definidos; extensa gama de estabelecimentos de saúde, que presta serviços de promoção, prevenção, diagnóstico, tratamento, gestão de casos, reabilitaçáo e cuidados paliativos; e Atenção Básica estruturada como primeiro nível de atenção e porta de entrada do sistema ${ }^{(6)}$.

Em 1970, nos Estados Unidos, surge o Gerenciamento de Casos em Saúde Mental, 
de forma contemporânea ao movimento de desospitalização, quando o fechamento de hospitais psiquiátricos resultou em elevadas taxas de reinternação, emergindo a necessidade da criação de estratégias mais efetivas para o tratamento comunitário. $\mathrm{O}$ método tem se mostrado efetivo para garantir a continuidade do tratamento para pacientes com transtornos mentais graves, tendo em vista a contraposiçãoao modelo tradicional hospitalocêntrico, o qualbusca tratar o paciente na comunidade e mantê-lo mais próximo do serviço de saúde ${ }^{(10)}$.

$\mathrm{Na}$ literatura brasileira, Mendes ${ }^{(7)}$ utiliza o termo Gestáo de Caso, e diz que esse consiste em um processo cooperativo, que se desenvolve entre um profissional gestor de caso e uma pessoa portadora de uma condição de saúde muito complexa e sua rede de suporte social para planejar, monitorar e avaliar opçóes de cuidados e de coordenação da atenção à saúde, de acordo com as necessidades da pessoa e com o objetivo de propiciar uma atenção de qualidade, humanizada, capaz de aumentar a capacidade funcional e de preservar autonomia individual e familiar.

Ainda segundo o autor, seus objetivos são advogar as necessidades e as expectativas de pessoas usuárias em situação especial, prover o serviço certo à pessoa certa, aumentar a qualidade do cuidado e diminuir a fragmentação da atenção à saúde. Esse processo envolve as seguintes etapas: seleção do caso; identificação do problema, elaboraçáo, implementação e monitoramento do plano de cuidado.

Cohen e Cesta ${ }^{(11)}$ referem que a enfermagem é constantemente escolhida para ser Gerente de Caso, tendo em vista que possui habilidades essenciais para o cumprimento desse papel, como por exemplo, a habilidadede realizar múltiplas açôes, a visão complexa do paciente, a atenção aos detalhes, a capacidade para tomada de decisão, a coordenação, a liderança e a comunicação.

Diante do desafio de cuidado a pacientes com transtornos mentais pela ESF, e a fim de colaborar na construção do conhecimento e na implantação efetiva da política pública definiu-se como objetivo deste estudo compreender os episódios de cuidado a mulheres com transtornos mentais atendidas na Estratégia da Saúde da Família.

\section{MÉTODO}

A pesquisa realizada foi de natureza qualitativa, por entender que esse tipo de pesquisa preocupa-se em estudar as sequências específicas dos comportamentos interpessoais, nas quais as açôes de cada pessoa são visualizadas em uma sequência interativa, que depende das açôes dos demais parceiros de interação. Estudando pequenas amostras, os estudos qualitativos privilegiam as regularidades e as singularidades nas análises ${ }^{(12)}$. O estudo foi aprovado pelo Comitê de Ética em Pesquisa em Seres Humanos da Universidade Federal de São João del Rei (parecer no 819.928).

$\mathrm{O}$ estudo foi realizado em Divinópolis, cidade do oeste de Minas Gerais (Brasil), que conta com vinte ESFs, 14 Centros de Saúde e dois Programas de Agentes Comunitários de Saúde, distribuídos em dez regiōes sanitárias. A coleta de dados foi realizada na Estratégia de Saúde da Família, localizada na região sanitária sudeste do município.

A coleta de dados ocorreu por meio da análise documental nos prontuários do serviço, arquivados na própria unidade. Esse tipo de pesquisa é relevante por proporcionar uma melhor visão do problema de pesquisa e hipóteses, que subsidiem a sua verificação por outras formas de pesquisa. Foram analisados 13 prontuários de atendimento a pacientes mulheres, pertencentes à ESF e vinculadas ao Centro de Atenção Psicossocial (CAPS III). A identificação dos prontuários foi realizada através de consulta ao Sistema de Informação em Saúde. Os dados foram coletados no período entre outubro de 2014 a março de 2015, a partir da análise documental dos prontuários do serviço. Para coleta de dados utilizou-se umformulário construído especificamente para a pesquisa, contendo dados sociodemográficos (sexo, estado civil, idade, escolaridade) e história dos episódios de cuidado (motivo da consulta, diagnóstico do 
problema percebido pelo profissional de saúde e intervenção resultante).

O referencial teórico utilizado para análise foi a Classificação Internacional da Atenção Primária (CIAP), sob a ótica do cuidado compartilhado. A CIAP trabalha com o conceito de episódio de cuidado e com a premissa de registrar três aspectos desse episódio (motivo da consulta, diagnóstico do problema e intervenção). $\mathrm{O}$ motivo da consulta é o elemento chave no registro, devendo ser classificado tal como expresso pelo paciente. É o sistema de classificação mais adequado para uso na Atenção Básica, e sua segunda edição (CIAP2) está organizada em 17 capítulos e sete componentes, e permite classificar os motivos da consulta e as respostas propostas pela equipe ${ }^{(13)}$.

O cuidado compartilhado é um processo permanente de pactuação, que oferece melhor qualidade e coordenação de cuidados através da interface: atenção primária-especialidade, e envolve múltiplos atores no processo de cuidado $^{(1)}$. Os componentes básicos para o modelo de cuidados compartilhados são: abordagem sistemática de generalista e especialista, com objetivos comuns de melhorar a saúde mental; modelo de tratamento coerente com as necessidades da população; abordagem clínica e monitoramento com revisão de especialistas; atenção para as necessidades da equipe; e supervisão para apoiar o desenvolvimento e a manutenção do modelo de tratamento e estrutura de governança clínica ${ }^{(14)}$.

\section{RESULTADOS}

Identifica-se que, em relação às fases de vida da mulher, a maioria encontra-se na idade reprodutiva (10 a 49 anos), tem baixa escolaridade, são casadas e com filhos. Entre as mulheres que apresentam morbidade clínica, verifica-se que a Hipertensão Arterial Sistêmica é doença mais comum. Dentre as morbidades psiquiátricas, verifica-se que o diagnóstico de ansiedade generalizada foi atribuído a mais da metade das mulheres, seguido do diagnóstico de esquizofrenia não especificada e episódio depressivo moderado (Quadro 1).

Quadro 1 - Perfil das mulheres com transtornos mentais atendidas na Atençâo Básica

\begin{tabular}{|c|c|c|c|c|}
\hline Caracterizaçáo & $\begin{array}{l}\text { Morbidade } \\
\text { Psiquiátrica }\end{array}$ & $\begin{array}{c}\text { Morbidade } \\
\text { Clínica }\end{array}$ & Atenção a Saúde & Terapêutica Medicamentosa \\
\hline $\begin{array}{l}\text { Mulher, } 31 \text { anos, } \\
\text { solteira, } 4 \text { filhos, ensino } \\
\text { médio incompleto }\end{array}$ & Ansiedade Generalizada & Asma/ Bronquite & $\begin{array}{c}\text { Atenção especializada em Saúde } \\
\text { Mental } \\
\text { Atençáo Especializada } \\
\text { Atençáo Diagnóstica } \\
\text { Atenção Básica } \\
\text { Atençáo Farmacêutica } \\
\end{array}$ & $\begin{array}{c}\text { Hipnótico/ sedativo } \\
\text { Ansiolítico } \\
\text { Antiepilético (3) } \\
\text { Antipsicótico }\end{array}$ \\
\hline $\begin{array}{c}\text { Mulher, } 62 \text { anos, } \\
\text { casada, } 3 \text { filhos, ensino } \\
\text { fundamental incompleto }\end{array}$ & $\begin{array}{l}\text { Esquizofrenia náo } \\
\text { especificada }\end{array}$ & Diabetes & $\begin{array}{c}\text { Atenção especializada em Saúde } \\
\text { Mental } \\
\text { Atenção Especializada } \\
\text { Atenção Diagnóstica } \\
\text { Atenção Básica } \\
\text { Atenção Hospitalar } \\
\text { Atenção Farmacêutica }\end{array}$ & $\begin{array}{c}\text { Antiagregante plaquetário } \\
\text { Antipsicótico } \\
\text { Ansiolítico } \\
\text { Antidiabético (2) }\end{array}$ \\
\hline $\begin{array}{l}\text { Mulher, } 55 \text { anos, } \\
\text { casada, } 3 \text { filhos, ensino } \\
\text { fundamental incompleto }\end{array}$ & Ansiedade Generalizada & HAS & $\begin{array}{c}\text { Atenção especializada em Saúde } \\
\text { Mental } \\
\text { Atenção Básica } \\
\text { Atenção Especializada } \\
\text { Atenção Farmacêutica } \\
\end{array}$ & $\begin{array}{c}\text { Antipsicótico } \\
\text { Antiepilético/ ansiolítico } \\
\text { Antidepressivo } \\
\text { Anti-hipertensivo (4) }\end{array}$ \\
\hline $\begin{array}{l}\text { Mulher, } 40 \text { anos, } \\
\text { casada, } 2 \text { filhos, ensino } \\
\text { fundamental incompleto }\end{array}$ & Depressão Moderada & Sem morbidade & $\begin{array}{c}\text { Atenção Diagnóstica } \\
\text { Atenção especializada em Saúde } \\
\text { Mental } \\
\text { Atenção Básica } \\
\text { Atenção Farmacêutica } \\
\end{array}$ & $\begin{array}{l}\text { Antidepressivo } \\
\text { Ansiolítico }\end{array}$ \\
\hline $\begin{array}{l}\text { Mulher, } 79 \text { anos, viúva, } \\
1 \text { filho, analfabeta }\end{array}$ & $\begin{array}{l}\text { Esquizofrenia náo } \\
\text { especificada }\end{array}$ & HAS & $\begin{array}{c}\text { Atenção Diagnóstica } \\
\text { Atenção especializada em Saúde } \\
\text { Mental } \\
\text { Atenção Básica } \\
\text { Atenção Farmacêutica }\end{array}$ & $\begin{array}{c}\text { Antiagregante plaquetário } \\
\text { Ansiolítico } \\
\text { Antidepressivo } \\
\text { Anti-hipertensivo }\end{array}$ \\
\hline
\end{tabular}




\begin{tabular}{|c|c|c|c|c|}
\hline $\begin{array}{l}\text { Mulher, } 25 \text { anos, casada, } \\
2 \text { filhos, ensino médio } \\
\text { incompleto }\end{array}$ & Ansiedade Generalizada & Sem morbidade & $\begin{array}{c}\text { Atenção especializada em Saúde } \\
\text { Mental } \\
\text { Atenção Básica } \\
\text { Atenção Especializada }\end{array}$ & Antidepressivo \\
\hline $\begin{array}{c}\text { Mulher, } 39 \text { anos, } \\
\text { casada, } 3 \text { filhos, ensino } \\
\text { fundamental incompleto }\end{array}$ & Ansiedade Generalizada & HAS & $\begin{array}{c}\text { Atenção especializada em Saúde } \\
\text { Mental } \\
\text { Atenção Básica } \\
\text { Atenção Especializada } \\
\text { Atenção Farmacêutica }\end{array}$ & $\begin{array}{l}\text { Ansiolítico } \\
\text { Antidepressivo } \\
\text { Antiulceroso }\end{array}$ \\
\hline $\begin{array}{l}\text { Mulher, } 48 \text { anos, solteira, } \\
\text { não possui filhos, ensino } \\
\text { fundamental incompleto }\end{array}$ & $\begin{array}{l}\text { Esquizofrenia náo } \\
\text { especificada }\end{array}$ & HAS & $\begin{array}{c}\text { Atenção especializada em Saúde } \\
\text { Mental } \\
\text { Atenção Diagnóstica } \\
\text { Atenção Básica } \\
\text { Atenção Hospitalar } \\
\text { Atenção Especializada } \\
\text { Atenção Farmacêutica }\end{array}$ & $\begin{array}{l}\text { Anti-hipertensivo } \\
\text { Antiulceroso } \\
\text { Ansiolítico } \\
\text { Antidepressivo (3) }\end{array}$ \\
\hline $\begin{array}{c}\text { Mulher, } 66 \text { anos, } \\
\text { viúva, } 6 \text { filhos, ensino } \\
\text { fundamental incompleto }\end{array}$ & $\begin{array}{l}\text { Esquizofrenia não } \\
\text { especificada }\end{array}$ & HAS & $\begin{array}{c}\text { Atenção especializada em Saúde } \\
\text { Mental } \\
\text { Atenção Farmacêutica } \\
\text { Atenção Especializada } \\
\text { Atenção Diagnóstica } \\
\text { Atenção Básica }\end{array}$ & $\begin{array}{c}\text { Antiagregante plaquetário } \\
\text { Antidepressivo } \\
\text { Ansiolítico } \\
\text { Antipsicótico } \\
\text { Antidislipidêmico } \\
\text { Anti-hipertensivo (2) }\end{array}$ \\
\hline $\begin{array}{c}\text { Mulher, } 51 \text { anos, } \\
\text { casada, } 2 \text { filhos, ensino } \\
\text { fundamental incompleto }\end{array}$ & Ansiedade Generalizada & Dislipidemia & $\begin{array}{c}\text { Atenção especializada em Saúde } \\
\text { Mental } \\
\text { Atenção Farmacêutica } \\
\text { Atenção Especializada } \\
\text { Atenção Básica }\end{array}$ & $\begin{array}{l}\text { Hipinótico/ sedativo } \\
\text { Antipsicótico } \\
\text { Antidislipidêmico }\end{array}$ \\
\hline $\begin{array}{c}\text { Mulher, } 43 \text { anos, } \\
\text { casada, } 3 \text { filhos, ensino } \\
\text { fundamental incompleto }\end{array}$ & Ansiedade Generalizada & Sem morbidade & $\begin{array}{c}\text { Atenção especializada em Saúde } \\
\text { Mental } \\
\text { Atenção Farmacêutica } \\
\text { Atenção Especializada } \\
\text { Atenção Básica }\end{array}$ & Ansiolítico (2) \\
\hline $\begin{array}{l}\text { Mulher, } 33 \text { anos, casada, } \\
1 \text { filho, ensino médio } \\
\text { completo }\end{array}$ & Depressão Moderada & Sem morbidade & $\begin{array}{c}\text { Atenção especializada em Saúde } \\
\text { Mental } \\
\text { Atenção Farmacêutica } \\
\text { Atenção Especializada } \\
\text { Atençáo Básica }\end{array}$ & $\begin{array}{l}\text { Antidepressivo } \\
\text { Antipsicótico (2) } \\
\text { Ansiolítico } \\
\text { Antialérgico }\end{array}$ \\
\hline $\begin{array}{l}\text { Mulher, } 23 \text { anos, } \\
\text { solteira, não possui } \\
\text { filhos, ensino médio } \\
\text { completo }\end{array}$ & Ansiedade Generalizada & Sem morbidade & $\begin{array}{c}\text { Atenção especializada em Saúde } \\
\text { Mental } \\
\text { Atençáo Especializada } \\
\text { Atenção Básica }\end{array}$ & Antidepressivo \\
\hline
\end{tabular}

Fonte: os autores.

Quanto aos pontos acessados na Rede de Atenção, percebe-se que todas as mulheres em estudo foram atendidas na Atenção Básica, Atenção especializada em Saúde mental. Além disso, todas tiveram atendimento em algum outro ponto da rede. Destes, os mais acessados foram Atenção Especializada e Farmacêutica. Embora todas as mulheres fizessem uso de algum tipo de medicação, evidenciou-se que uma pequena parte não acessou este ponto da Atenção.

Verificou-se, também, que a maior parte das mulheres que foram atendidas na Atenção Especializada, possui um diagnóstico clínico associado, em sua maioria, à Hipertensão Arterial Sistêmica; e que os pontos da rede menos acessados foram a Atenção Hospitalar e a Atenção Diagnóstica.
Quanto a medicação, havia registro de prescrição de medicamentos em todos os prontuários, sendo a classe dos ansiolíticos, antidepressivos e antipsicóticos os tipos de medicamento mais prescritos, respectivamente. Dentre as mulheres com diagnósticos de ansiedade generalizada, todas foram indicadas a fazer uso de ansiolítico e/ou antidepressivo. Para todas as mulheres com comorbidade clínica associada, havia de registro de prescriçáo para tal fim, como os antihipertensivos, antidiabéticos e antidislipidêmicos.

Quanto ao tipo de atendimento na $A B$, os atendimentos encontrados foram: consulta médica agendada, consulta médica livre demanda, consulta de enfermagem agendada, consulta de enfermagem livre demanda, consulta de psicologia e visita domiciliar (Quadro 2). 
Quadro 2 - Caracterização do atendimento às mulheres com transtornos mentais atendidas na Atenção Básica

\begin{tabular}{|c|c|c|c|}
\hline Caracterizaçáoo & $\begin{array}{l}\text { Número médio de } \\
\text { atendimento na Atençáa } \\
\text { Básica por mês }\end{array}$ & $\begin{array}{l}\text { Cuidado profissional na } \\
\text { Atençáo Básica }\end{array}$ & Caraterizaçáo do atendimento \\
\hline $\begin{array}{l}\text { Mulher, } 31 \text { anos, solteira, } 4 \\
\text { filhos, ensino médio incompleto }\end{array}$ & 1,1 & $\begin{array}{l}\text { Cuidado médico - } 6 \\
\text { Cuidado de enfermagem - } 3 \\
\text { Cuidado de psicologia - } 7\end{array}$ & $\begin{array}{c}\text { Demanda espontânea (médico) - } 04 \\
\text { Demanda espontânea (enfermagem) - } 02 \\
\text { Consulta médica - 02 } \\
\text { Consulta de enfermagem - } 01 \\
\text { Visita domiciliar - } 01 \\
\text { Consulta de psicologia - } 06\end{array}$ \\
\hline $\begin{array}{l}\text { Mulher, } 62 \text { anos, casada, } 3 \text { filhos, } \\
\text { ensino fundamental incompleto }\end{array}$ & 0,5 & $\begin{array}{l}\text { Cuidado médico - } 29 \\
\text { Cuidado de enfermagem - } 5\end{array}$ & $\begin{array}{c}\text { Demanda espontânea (médico) - } 12 \\
\text { Demanda espontânea (enfermagem) - } 03 \\
\text { Consulta médica - } 17 \\
\text { Consulta de enfermagem - } 02\end{array}$ \\
\hline $\begin{array}{l}\text { Mulher, } 55 \text { anos, casada, } 3 \text { filhos, } \\
\text { ensino fundamental incompleto }\end{array}$ & 0,5 & $\begin{array}{l}\text { Cuidado médico - } 18 \\
\text { Cuidado de enfermagem - } 6 \\
\text { Cuidado de psicologia - } 14\end{array}$ & $\begin{array}{c}\text { Demanda espontânea (médico) - } 17 \\
\text { Demanda espontânea (enfermagem) - } 02 \\
\text { Visita domiciliar médica - } 01 \\
\text { Visita domiciliar enfermagem - } 04 \\
\text { Visita domiciliar Psicologia - } 01 \\
\text { Consulta de psicologia - } 13\end{array}$ \\
\hline $\begin{array}{l}\text { Mulher, } 40 \text { anos, casada, } 2 \text { filhos, } \\
\text { ensino fundamental incompleto }\end{array}$ & 0,6 & $\begin{array}{l}\text { Cuidado médico - } 5 \\
\text { Cuidado de enfermagem - } 11 \\
\text { Cuidado de psicologia - } 3\end{array}$ & $\begin{array}{c}\text { Demanda espontânea (médico) - } 03 \\
\text { Demanda espontânea (enfermagem) - } 09 \\
\text { Consulta médica - } 02 \\
\text { Consulta de enfermagem - } 02 \\
\text { Consulta de psicologia - } 03\end{array}$ \\
\hline $\begin{array}{l}\text { Mulher, } 79 \text { anos, viúva, } 1 \text { filho, } \\
\text { analfabeta }\end{array}$ & 0,6 & $\begin{array}{l}\text { Cuidado médico - } 33 \\
\text { Cuidado de enfermagem - } 6 \\
\text { Cuidado de psicologia -1 }\end{array}$ & $\begin{array}{c}\text { Demanda espontânea (médico) - } 15 \\
\text { Demanda espontânea (enfermagem) - } 04 \\
\text { Consulta médica - } 18 \\
\text { Consulta de enfermagem - } 01 \\
\text { Visita domiciliar enfermagem - } 01 \\
\text { Consulta de psicologia - } 01\end{array}$ \\
\hline $\begin{array}{l}\text { Mulher, } 25 \text { anos, casada, } 2 \text { filhos, } \\
\text { ensino médio incompleto }\end{array}$ & 0,4 & $\begin{array}{l}\text { Cuidado médico - } 11 \\
\text { Cuidado de enfermagem - } 9\end{array}$ & $\begin{array}{c}\text { Demanda espontânea (médico) - } 09 \\
\text { Demanda espontânea (enfermagem) - } 07 \\
\text { Consulta médica - } 02 \\
\text { Consulta de enfermagem - } 02\end{array}$ \\
\hline $\begin{array}{l}\text { Mulher, } 39 \text { anos, casada, } 3 \text { filhos, } \\
\text { ensino fundamental incompleto }\end{array}$ & 0,5 & $\begin{array}{l}\text { Cuidado médico - } 25 \\
\text { Cuidado de enfermagem - } 4\end{array}$ & $\begin{array}{l}\text { Demanda espontânea (médico) - } 12 \\
\text { Demanda espontânea (enfermagem) - } 0 \\
\text { Consulta médica - 13 } \\
\text { Consulta de enfermagem - } 04\end{array}$ \\
\hline $\begin{array}{l}\text { Mulher, } 48 \text { anos, solteira, não } \\
\text { possui filhos, ensino fundamental } \\
\text { incompleto }\end{array}$ & 1,1 & $\begin{array}{l}\text { Cuidado médico - } 13 \\
\text { Cuidado de enfermagem - } 9 \\
\text { Cuidado de psicologia - } 22\end{array}$ & $\begin{array}{c}\text { Demanda Espontânea - } 07 \\
\text { Demanda espontânea (enfermagem) - } 08 \\
\text { Consulta médica - 06 } \\
\text { Consulta de enfermagem - } 01 \\
\text { Consulta de psicologia - } 22\end{array}$ \\
\hline $\begin{array}{l}\text { Mulher, } 66 \text { anos, viúva, } 6 \text { filhos, } \\
\text { ensino fundamental incompleto }\end{array}$ & 0,3 & $\begin{array}{l}\text { Cuidado médico - } 20 \\
\text { Cuidado de enfermagem - } 5\end{array}$ & $\begin{array}{c}\text { Demanda espontânea (médico) - } 09 \\
\text { Demanda espontânea (enfermagem) - } 04 \\
\text { Consulta médica - } 11 \\
\text { Consulta de enfermagem - } 01\end{array}$ \\
\hline $\begin{array}{l}\text { Mulher, } 51 \text { anos, casada, } 2 \text { filhos, } \\
\text { ensino fundamental incompleto }\end{array}$ & 0,2 & $\begin{array}{c}\text { Cuidado médico - } 3 \\
\text { Cuidado de enfermagem - } 10\end{array}$ & $\begin{array}{c}\text { Demanda espontânea (médico) - } 03 \\
\text { Demanda espontânea (enfermagem) - } 03 \\
\text { Consulta médica - 0 } \\
\text { Consulta de enfermagem - } 03 \\
\text { Visita domiciliar enfermagem - } 04\end{array}$ \\
\hline $\begin{array}{l}\text { Mulher, } 43 \text { anos, casada, } 3 \text { filhos, } \\
\text { ensino fundamental incompleto }\end{array}$ & 0,3 & $\begin{array}{l}\text { Cuidado médico - } 21 \\
\text { Cuidado de enfermagem - } 1 \\
\text { Cuidado de psicologia - } 1\end{array}$ & $\begin{array}{c}\text { Demanda Espontânea - } 13 \\
\text { Demanda espontânea (enfermagem) - } 01 \\
\text { Consulta médica - } 08 \\
\text { Consulta de enfermagem - } 0 \\
\text { Consulta de psicologia - } 01\end{array}$ \\
\hline $\begin{array}{l}\text { Mulher, } 33 \text { anos, casada, } 1 \text { filho, } \\
\text { ensino médio completo }\end{array}$ & 0,8 & $\begin{array}{l}\text { Cuidado médico - } 11 \\
\text { Cuidado de enfermagem - } 14\end{array}$ & $\begin{array}{c}\text { Demanda espontânea (médico) - } 09 \\
\text { Demanda espontânea (enfermagem) - } 07 \\
\text { Consulta médica - } 02 \\
\text { Consulta de enfermagem - } 07\end{array}$ \\
\hline $\begin{array}{l}\text { Mulher, } 23 \text { anos, solteira, não } \\
\text { possui filhos, ensino médio } \\
\text { completo }\end{array}$ & 0,2 & $\begin{array}{l}\text { Cuidado médico - } 5 \\
\text { Cuidado de enfermagem - } 6 \\
\text { Cuidado de psicologia - } 2\end{array}$ & $\begin{array}{c}\text { Demanda Espontânea (médico) - } 02 \\
\text { Demanda espontânea (enfermagem) - } 03 \\
\text { Consulta médica - } 03 \\
\text { Consulta de enfermagem - } 03 \\
\text { Consulta de psicologia - } 02\end{array}$ \\
\hline
\end{tabular}

Fonte: os autores. 
O tipo de atendimento de livre demanda foi 1,4 vezes maior que o atendimento por agendamento; e ainda, as mulheres que mais foram ao atendimento por livre demanda são, em sua maioria, aquelas com diagnóstico psiquiátrico de Transtorno de Ansiedade Generalizada (TAG), e/ou com diagnóstico clínico associado, assim como as que foram atendidas na consulta de psicologia e demandaram, assim, mais cuidado da equipe multiprofissional.

Dentre as mulheres que foram atendidas por consulta médica e de enfermagem, previamente agendada, a maioria delas possui morbidade clínica associada e outro diagnóstico psiquiátrico não relacionado ao TAG. Dentre as que foram atendidas a domicílio, todas apresentam morbidade clínica associada. A grande maioria apresentou diagnóstico de TAG.

Quanto aos cuidados oferecidos na atenção básica, verificou-se que o cuidado oferecido pelo profissional médico foi 2,3 vezes maior que o cuidado oferecido pelo profissional de enfermagem e 4,2 vezes maior que o cuidado oferecido pelo profissional de psicologia; E o cuidado oferecido pelo profissional de enfermagem foi 1,7 vezes maior que o cuidado oferecido pelo profissional de psicologia.

\section{DISCUSSÃO}

Segundo Mendes $^{(7)}$, um dos objetivos fundamentais dos sistemas de atenção à saúde é a qualidade. Desse modo, o autor propóe um sistema integrado, pela implantação de uma Rede de Atenção à Saúde (RAS), que tem como pilar a Atenção Primária à Saúde. Segundo o autor, a estrutura operacional das RAS é composta por cinco componentes: a APS; os pontos de atenção à saúde secundários e terciários; os sistemas de apoio (diagnóstico e terapêutico, sistema de assistência farmacêutica e sistema de informação em saúde); os sistemas logísticos (cartão de identificação das pessoas usuárias, prontuário clínico, sistemas de acesso regulado à atenção e sistemas de transporte em saúde) e o sistema de governança.
Embora a atenção básica seja a porta de entrada preferencial dos indivíduos na rede de saúde e o dispositivo ordenador da rede de cuidado, ficou evidente que os principais atendimentos registrados (encaminhamentos, prescrição medicamentosa, coleta de exame, acolhimento, etc.), não evidenciaram a continuidade do cuidado, pois a não existência de uma contrarreferências faze com que as usuárias fiquem abandonadas a si mesmas, dificultando assim a promoção de vínculo e acolhimento de suas famílias e necessidades.

Neste sentido, Ribeiro ${ }^{(15)}$ enfatiza que a Estratégia de Saúde da Família, por ser um dos equipamentos mais próximos à comunidade, tem o potencial de auxiliar na articulação com os diversos dispositivos da rede, a fim de prevenir doenças e promover saúde. Tanaka e Ribeiro ${ }^{(16)}$ reafirmam que, na Atenção Básica, há o potencial de detectar as queixas relativas ao sofrimento psíquico e prover uma escuta qualificada para a problemática, oferecendo tratamento no território ou encaminhando para serviços especializados, justificando assim a impotência de uma execução correta e eficaz da rede de cuidado.

O Gerenciamento de Caso (GC) é uma questão importante na enfermagem contemporânea, podendo ser implementado em diferentes áreas da enfermagem, e seu conceito é proveniente do termo "qualidade do cuidado", quando se refere mais precisamente à psiquiatria e à saúde mental ${ }^{(17)}$.

De acordo com Henderson et al. ${ }^{(18)}$, gerenciar casos em saúde mental consiste em avaliar as necessidades individuais e desenvolver um plano de cuidados para atender a essas necessidades. Para tal, é preciso um guia de cuidados multidisciplinares e um gerenciador de casos, sendo o guia uma lista de problemas do paciente com expectativas de resultados e uma lista de intervenções prescritas por um longo tempo; e o gerenciador um profissional que assegure o cuidado a este paciente ${ }^{(14)}$.

A gestão de caso é eficaz quando as condiçôes crônicas são múltiplas e complexas, havendo um nível considerável de interdependência entre os 
profissionais, incertezas em relação às intervenções clínicas e a necessidade de utilização dos vários pontos de atenção do sistema de saúde durante o processo de tratamento e reabilitação. Nessas situaçóes, é necessário viabilizar a comunicação pessoal entre os profissionais envolvidos no manejo do caso ${ }^{(19)}$.

\section{CONSIDERAÇÕES FINAIS}

A Atenção Básica através da ESF é a principal forma de entrada dos usuários, com transtornos mentais, ao sistema de saúde e ao centro de comunicação, com todas as Redes de Atenção à Saúde. Essas, por sua vez, possuem como objetivo melhorar a qualidade da atenção, oferecendo serviços de saúde contínuo aos pacientes. Apesar disso, algumas vezes, os usuários desses serviços iniciam um atendimento que não possui a continuidade do cuidado. Desse modo, fica evidente a importância do desenvolvimento de estratégias mais efetivas, como o Gerenciamento de casos, a fim de promover a continuidade do acompanhamento e tratamento dos pacientes com transtornos mentais, evitando internações e possibilitando a manutenção do cuidado em seu cotidiano.

\section{REFERÊNCIAS}

1. Brasil. Ministério da Saúde. Secretaria deAtenção à Saúde. Departamento de Atenção Básica. Saúde mental / Ministério da Saúde, Secretaria de Atenção à Saúde, Departamento de Atenção Básica, Departamento de Açôes Programáticas Estratégicas. - Brasília : Ministério da Saúde, 2013. 176 p. : il. (Cadernos de Atenção Básica, n. 34)

2. Brasil. A Reforma Psiquiátrica Brasileira e a Polítca de Saúde Mental. Disponível em <http://www.ccs.saude.gov.br/vpc/reforma. html. Acesso em 13/05/2014>.

3. CezarPK,RodriguesPM,ArpiniDM.APsicologia na Estratégia de Saúde da Família: Vivências da
Residência Multiprofissional. Psicol. cienc. prof. 2015; 35(1):211-224.

4. Correia VR, Barros S, Colvero, LA. Saúde mental na atenção básica: prática da equipe de saúde da família. Rev. esc. enferm. 2011; 45(6):1501-1506.

5. Estevam MC, et al.. Convivendo com transtorno mental: perspectiva de familiares sobre Atenção Básica. Rev. esc. enferm. USP [online]. 2011; 45, (3):679-686.

6. Brasil. Ministério da Saúde. Secretaria de Gestão do Trabalho e da Educação na Saúde. Secretaria de Atenção à Saúde. Edital no 14 , de 08 de março de 2013. Edital para o Programa de Educaçáo pelo Trabalho para a Saúde - PETSaúde/Redes de Atenção à Saúde (PET-Saúde/Redes). Brasília: Diário Oficial da Uniāo; 11 Mar 2013

7. Mendes EV. As redes de atenção à saúde. Brasília, DF: Organização Pan-Americana da Saúde, 2011.

8. World Health Organization. Who report on the global tobacco epidemic, 2008 - the MPOWER package. Geneva: World Health Organization 2008.

9. Rosen R, Ham C. Atención integrada: enseñanzas de evidencia y experiencia, informe delseminario Anual de Salud 2008 Sir Roger Banninster. Revista de InnovaciónSanitaria y Atención Integrada 2008; 1:2.

10. Marshall M, Gray A, Lockwood A, Green R. Withdrawn: case management for people with severe mental disorders. Cochrane Database System Rev [internet]. 2011; (4):CD000050. Disponível em: http://www.update-software. com/BCP/WileyPDF/EN/CD000050.pdf.

11. Cohen EL, Cesta TG, editoras. Nursing case management: from essentials to advanced practice applications. $4^{\mathrm{a}}$ ed. Philadelphia: Elsevier; 2005.

12. Wendt NC, Crepaldi MA. A utilização do genograma como instrumento de coleta de dados em pesquisa qualitativa. Psicologia: Reflexão e Crítica.2008, 21:302-310. 
13. Okkes IM, Oskam SK, Lamberts H.The probability of specific diagnoses for patients presenting with common symptoms to Dutch family physicians. J Fam Pract.2002;51:31-6

14. Kelly PJ, Deane FP, King R, Katanzis N, Crowe TP. A taxonomy for homework used by mental health case managers when working with individuals diagnosed with severe mental illness. Communy Mental Health J. 2007; 43(6):565-80.

15. Ribeiro KSQS. Redes sociais e educação popular: aproximaçáo teórica e mudanças na prática de educação popular em saúde. In: Anais do V Colóquio Internacional Paulo Freire; 2005 set. 19-22; Recife, PE, Brasil.

16. Tanaka OY, Ribeiro EL. Açóes de saúde mental na atenção básica: caminho para ampliação da integralidade da atenção. CienSaude Colet. 2009; 14(2):477-486.

17. Lee D, Mackenzie A, Dudley-Brown S, Chin T. Case management: a review of the definitions and practice. J. Adv. Nurs. 1998; 2(7):933-9.

18. Henderson J, Willis E, Walter B, Toffoli L. Community Mental Health Nursing: keeping pace with care delivery? Int J Mental Health Nurs. 2008; 17:162-70.

19. Organização Pan-Americana da Saúde. Plano de Desenvolvimento Institucional da OPAS/ OMS no Brasil 2010-2011/ Organização PanAmericana da Saúde. Brasília: Organização PanAmericana da Saúde, 2010. 